Pesq. Vet. Bras. 35(4):319-323, abril 2015 DOI: $10.1590 / \mathrm{S} 0100-736 \mathrm{X} 2015000400001$

\title{
Estudo eletrocardiográfico de éguas da raça Crioula ${ }^{1}$
}

\author{
João P.E. Pascon²*, Fernanda P. Santos ${ }^{2}$, Deyverson T.P. Pereira ${ }^{2}$, Maria L.A. Mistieri ${ }^{2}$ \\ e Fabrício D. Mozzaquatro ${ }^{2}$
}

\begin{abstract}
Pascon J.P.E., Santos F.P., Pereira D.T.P., Mistieri M.L.A. \& Mozzaquatro F.D. 2015. [Electrocardiographic study of the Creole breed mares.] Estudo eletrocardiográfico de éguas da raça Crioula. Pesquisa Veterinária Brasileira 35(4):319-323. Serviço de Cardiologia Veterinária, Curso de Medicina Veterinária, Universidade Federal do Pampa, Rodovia BR-472 Km 592, Uruguaiana, RS 97500-970, Brazil. E-mail: joaopascon@unipampa.edu.br

The electrocardiography is an indispensable diagnostic tool to detect heart arrhythmias and electrical conductions disturbances in equines, as well as on prognosis determination of heart diseases, athletical performance, efficiency of training, and on electrolytic disturbance evaluation. However, electrocardiographic variables can be influenced by several factors such as age, gender, breed and phenotypic characteristics. Therefore the establishment of the characteristics of normality for different breed and phases of development is fundamental. Creole breed were firstly introduced to the American continent more than four centuries ago and it descends from horses of Iberian Peninsula. The breed is a result of natural selection that configured resistance and unique physical attributes. The present study aimed to analyze and compare the electrocardiographic parameters of Creole females in different ages, and verify the possibly influence of pregnancy on it. Digital electrocardiographic evaluation on apex-base lead was performed on 84 healthy creole mares (34 pregnant and 50 non-pregnant). The electrocardiograms were divided in groups concerning the age as G1 (until 4 years old), G2 (from 5 to 9 years old) and G3 (over 10 years old). No physiological or pathological heart arrhythmias and electrical conduction disturbances were seen in all examined mares. Sinus tachycardia, bifid P waves, rS configuration of QRS and biphasic T waves were the predominant patterns in all evaluated groups. Only the average of QRS duration of G1 females was significant superior $(110,65 \pm 8,49)$ $(p=0,0002)$ when compared with G2 $(101,98 \pm 10,02)$ and G3 $(100,92 \pm 10,72)$. The autonomic variables (ITV, average NN and SDNN) were lower in pregnant mares comparing to non-pregnant mares, suggesting a greater sympathetic autonomic system tonus and/or decrease of parasympathetic tonus on pregnant mares. In conclusion, the age affect only the QRS duration, and the pregnant condition was capable to decrease the time domain heart rate variability indexes with maybe influence the electrocardiographic evaluation of enrolled Creole mares.
\end{abstract}

INDEX TERMS: Electrocardiogram, equidae, ECG, cardiology, apex-base lead.

RESUMO.- A eletrocardiografia constitui ferramenta indispensável no diagnóstico de arritmias e distúrbios de condução elétrica do coração de equinos, bem como na determinação do prognóstico de cardiopatias, do desempenho atlético, da eficiência do treinamento, além de sugerir dis-

\footnotetext{
${ }^{1}$ Recebido em 4 de fevereiro de 2014.

Aceito para publicação em 22 de dezembro de 2014.

${ }^{2}$ Curso de Medicina Veterinária, Universidade Federal do Pampa (Unipampa), Rodovia BR472 Km 592, Uruguaiana, RS 97500-970, Brasil. *Autor para correspondência: joaopascon@unipampa.edu.br
}

túrbios eletrolíticos. No entanto, as variáveis eletrocardiográficas em equinos podem sofrer influência de diversos fatores como a idade, sexo, raça e constituição morfofuncional, dentre outas, tornando-se necessário conhecer as características de normalidade para as diferentes raças e fases do desenvolvimento. Descendentes dos cavalos da Península Ibérica, a raça Crioula foi trazida ao continente americano há mais de quatro séculos, resultando em características físicas e de resistência únicas, dada por sua seleção natural. Desta forma, objetivou-se com o presente trabalho avaliar e comparar os aspectos eletrocardiográficos 
de fêmeas da raça Crioula, em diferentes idades, bem como avaliar possíveis alterações eletrocardiográficas secundárias a prenhes. Para tanto, 84 éguas hígidas (34 prenhes e 50 não prenhes) da raça Crioula foram submetidas à avaliação eletrocardiográfica digital na derivação ápice-base, e os registros eletrocardiográficos subdivididos quanto à idade em G1 (até 4 anos), G2 (5 a 9 anos), G3 (acima de 10 anos). Não foram observadas arritmias cardíacas fisiológicas ou patológicas e distúrbios de condução elétrica do coração nas 84 éguas. Houve predomino de taquicardia sinusal, ondas $\mathrm{P}$ bífidas, complexos QRS do tipo rS e ondas T bifásicas em todos os grupos. Apenas a duração média do complexo QRS foi superior no grupo G1 $(110,65 \pm 8,49)$ quando comparadas aos grupos G2 $(101,98 \pm 10,02)$ e G3 $(100,92 \pm 10,72)$. As variáveis autonômicas mensuradas (ITV, NNmédio e SDNN) foram inferiores nas éguas prenhes em relação às não prenhes, sugerindo maior participação do sistema nervoso autônomo simpático e ou menor participação parassimpática. Conclui-se, portanto, que a idade influenciou apenas na duração do complexo QRS , e que a prenhes foi capaz de diminuir as variáveis de variabilidade da frequência cardíaca no domínio do tempo e, possivelmente, influenciar na avaliação eletrocardiográfica das éguas Crioulas aqui testadas.

TERMOS DE INDEXAÇÃO: Eletrocardiograma, equídeos, ECG, cardiologia, derivação ápice-base.

\section{INTRODUÇÃO}

O eletrocardiograma (ECG) representa o registro gráfico da atividade elétrica cardíaca, captado na superfície corpórea por meio da diferença de potencial elétrico gerada por eletrodos (derivações), indicado para a detecção de arritmias, distúrbios de formação e condução elétrica do coração (Fernandes et al. 2004). Em equinos, a avaliação eletrocardiográfica é também empregada na determinação do desempenho atlético e eficácia do treinamento (Lightowler et al. 2004, Bello et al. 2012), detecção de distúrbios eletrolíticos (Babusci \& López 2006) e acompanhamento de cardiopatias (Marr \& Bowen 2010).

0 exame é pouco oneroso, não-invasivo e exequível a campo, dependente apenas do equipamento e da existência de profissionais capacitados à sua realização e interpretação (Robertson 1990). Entretanto, os parâmetros eletrocardiográficos podem apresentar variação entre as diferentes raças e sexo de equinos, bem como entre as fases do desenvolvimento, exigindo a padronização racial, sexual e etária dos valores eletrocardiográficos de normalidade (Fernandes et al. 2004, Diniz et al. 2008), os quais ainda são limitados na raça Crioula (Corredor et al. 2005, Schade et al. 2014).

A raça Crioula descende de cavalos espanhóis, introduzidos e registrados no Sul do Brasil a partir de 1932, com significativa expansão nas últimas três décadas. Ao longo da adaptação e seleção natural sofrida pela raça, incorporou-se características próprias de rusticidade e resistência, conferindo a estes animais a capacidade de enfrentar as diversidades climáticas e a sua manutenção em campos nativos. Devido a estas características, a raça crioula apresenta boa desenvoltura no trabalho rural e em competições esportivas, assumindo assim grande importância no cenário econômico, cultural e social (ABCCC 2014).

Desta forma, objetivou-se com o presente estudo avaliar e comparar as características eletrocardiográficas de éguas da raça Crioula do município de Uruguiana-RS, em diferentes faixas etárias, bem como avaliar possíveis alterações eletrocardiográficas secundárias a prenhes.

\section{MATERIAL E METÓDOS}

A avaliação eletrocardiográfica computadorizada digital foi realizada em 84 éguas hígidas (34 prenhes e 50 não prenhes) da raça Crioula, devidamente registradas na Associação Brasileira de Criadores de Cavalos Crioulos (ABCCC), provenientes de propriedades rurais do município de Uruguaiana-RS. As variáveis eletrocardiográficas foram avaliadas em três faixas etárias , sendo a primeira composta por 40 éguas com idade até quatro anos (G1), enquanto a segunda (G2) formada por 11 éguas entre cinco e nove anos e a terceira faixa (G3) contendo 33 éguas com idade superior à 10 anos.

Para realização do exame eletrocardiográfico, as éguas foram mantidas em tronco de contenção apropriado para espécie, em posição ortostática, sem o uso de tranquilizantes ou sedativos. 0 registro eletrocardiográfico foi obtido por meio de eletrocardiógrafo computadorizado digital (módulo de ECGPC veterinário, TEB $^{\circledR}$, Brasil), na derivação bipolar ápice-base (Patteson 1996, Loon \& Patteson 2010), e interpretado com auxílio de software específico (software de análise ECGPC veterinário versão 2.27, TEB $^{\circledR}$, Brasil) na sensibilidade $\mathrm{N}(1 \mathrm{~cm}=1 \mathrm{mV})$ e velocidade de $25 \mathrm{~mm} / \mathrm{s}$.

Os parâmetros eletrocardiográficos foram analisados e comparados entre as diferentes idades, de forma descritiva (frequência de ocorrência) para as variáveis qualitativas de ritmo, conformação das ondas P e complexo QRS, além da polaridade das ondas T. Por meio da análise de variância seguida do teste Tukey ( $p>0,05)$, para comparação entre os grupos G1, G2 e G3, foram analisadas as variáveis quantitativas médias de FC, duração das ondas P, intervalos PR, complexo QRS, intervalo QT, média de 15 intervalos RR normais (NN médio), desvio padrão de 15 intervalos RR normais (SDNN) e índice de tônus vagal (ITV), em milissegundos, além da amplitude das ondas P, R e S. Os indicadores autonômicos NN médio, SDNN e ITV (Tárraga 2002) foram calculados no último minuto de registro ECG, utilizando-se 15 intervalos RR consecutivos, de complexos normais.

0 presente estudo foi submetido e aprovado pelo Comitê de Ética no Uso de Animais da Universidade Federal do Pampa (Parecer 021/2012).

\section{RESULTADOS E DISCUSSÃO}

Os ritmos (Quadro 1), durações e amplitudes das ondas e intervalos eletrocardiográficos avaliados encontram-se dispostos, quanto à raça (total de éguas) e idades (G1, G2 e G3), nos Quadros 2 e 3, respectivamente. Na avaliação do ritmo cardíaco, foi evidenciado predomínio de taquicardia sinusal (TS) em todas as idades (G1, G2 e G3), seguido por ritmo sinusal e arritmia sinusal respiratória em apenas uma égua do grupo G1. No entanto, estudos eletrocardiográficos envolvendo equinos da raça Crioula, machos e fêmeas, de diferentes idades, demonstraram predomínio do ritmo sinusal (Corredor et al. 2005, Schade et al. 2014), seguido por TS e arritmia sinusal respiratória (Schade et al. 2014). Embora muitos fatores possam estar envolvidos na 
elevação da frequência cardíaca (FC) das éguas estudadas, resultando em maior prevalência de TS, os autores acreditam que esta diferença possa estar relacionada há três fatores principais.

0 primeiro trata de questão conceitual relacionada à FC limítrofe (máxima) utilizada para determinação da TS. Em sua concepção, a TS é determinada a partir da origem sinusal dos complexos eletrocardiográficos, aliada à frequência de despolarização superior ao limite de FC normal para a espécie (FC limítrofe). No presente estudo, a FC limítrofe adotada (46bpm) considerou $40 \mathrm{bpm}$ como valor basal de normalidade para espécie equina (Feitosa 2004), acrescido de seis bpm pelo fator manipulação (Craig \& Nunan 1998). Embora a metodologia dos trabalhos citados não determine qual foi a $\mathrm{FC}$ limítrofe utilizada para esta

Quadro 1. Frequência (\%) dos ritmos cardíacos verificados das éguas da raça Crioula, em diferentes idades (G1, G2 e G3)

\begin{tabular}{lccc}
\hline Grupos & ASR & RS & TS \\
\hline G1 $(\mathrm{n}=40)$ & 2,5 & 15,0 & 82,5 \\
G2 $(\mathrm{n}=11)$ & 0 & 18,2 & 81,8 \\
G3 $(\mathrm{n}=33)$ & 0 & 12,1 & 87,9
\end{tabular}

$\overline{\mathrm{ASR}}=$ arritmia sinusal respiratória, $\mathrm{RS}=$ ritmo sinusal, $\mathrm{TS}=$ taquicardia sinusal.

\section{Quadro 2. Variáveis eletrocardiográficas médias e} desvios-padrão de 84 éguas da raça Crioula

\begin{tabular}{lc}
\hline \multicolumn{1}{c}{ Variável } & Média geral \\
\hline Frequência Cardíaca (bpm) & $53,22 \pm 8,65$ \\
Duração onda P (ms) & $110,10 \pm 21,42$ \\
Amplitude onda P (mV) & $0,17 \pm 0,05$ \\
Intervalo PR (ms) & $240,10 \pm 40,28$ \\
Complexo QRS (ms) & $105,80 \pm 10,62$ \\
Amplitude R (mV) & $0,16 \pm 0,11$ \\
Amplitude S (mV) & $0,12 \pm 0,03$ \\
Intervalo QT (ms) & $199,70 \pm 29,65$ \\
ITV & $3,37 \pm 0,58$ \\
NN médio (ms) & $1133,00 \pm 179,00$ \\
SDNN (ms) & $60,77 \pm 43,66$ \\
Peso (kg) & $498,60 \pm 76,41$
\end{tabular}

$\overline{\mathrm{Bpm}}=$ batimentos por minuto, $\mathrm{ms}=$ milissegundo, $\mathrm{mV}=$ milivolt, $\mathrm{ITV}=$ índice de tônus vagal, NNmédio = média de 15 intervalos R-R normais consecutivos, SDNN = desvio padrão de 15 intervalos R-R normais consecutivos. finalidade, pudemos observar, para a raça Crioula, que a FC média de éguas prenhas $(52,926 \pm 10,35 \mathrm{bpm})$, equinos machos e fêmeas entre 1 e 2 anos $(50,6 \pm 12,023 \mathrm{bpm})$ e com mais de 11 anos $(48,375 \pm 12,419 \mathrm{bpm}$ ) (Schade et al. 2014), assim como éguas prenhas com menos de cinco anos $(69,25 \pm 13,04 \mathrm{bpm})$ e machos inteiros com menos de cinco anos (48,75 $\pm 11,10 \mathrm{bpm}$ ) (Corredor et al. 2005), esteve acima da FC limítrofe de $46 \mathrm{bpm}$ utilizada no presente estudo, o que caracterizaria a TS.

0 segundo aspecto a ser considerado refere-se a possível diferença entre o grau de domesticação dos animais estudados e sua influência sobre a FC. Schade et al. (2014) observaram FC média superior em cavalos crioulos não domados quando comparados aos domados (treinados e não treinados). Os autores atribuíram este efeito à maior excitação apresentada pelos animais não domados durante a execução do exame. De forma semelhante, as 84 éguas avaliadas no presente estudo não eram domadas, sendo mantidas em sistema extensivo de criação, com pouca manipulação humana, o que pode ter resultado em maior excitação e elevação da FC.

Outrossim, a alta porcentagem de fêmeas prenhe (59\%) é apontada como possível fator capaz de elevar a FC das éguas aqui estudadas, a exemplo do também reportado por Schade et al. (2014). Possivelmente, as adaptações orgânicas resultantes da prenhes sejam responsáveis pelo aumento da velocidade de condução elétrica cardíaca, principalmente pela elevação dos níveis séricos de hormônios tireoidianos e cortisol observados durante a prenhes (Teixeira et al. 2008). Isso resulta em maior taxa metabólica e sensibilidade adrenérgica do miocárdio, capaz de aumentar a velocidade de condução elétrica e reduzir o tempo de despolarização ventricular, por ação direta no miocárdio ou por meio da ativação do sistema nervoso autônomo simpático.

Arritmias e distúrbios de condução elétrica do coração como bradicardia sinusal, bloqueios atrioventriculares de I e II graus, bloqueio sinusal ou sinus arrest (Loon \& Patteson 2010, Diniz et al. 2011) e complexos atriais e/ou ventriculares prematuros isolados (Dumont et al. 2010, Diniz et al. 2011) são frequente mente detectados em equinos saudáveis de diversas raças, sem significado clínico. No entanto,

Quadro 3. Variáveis eletrocardiográficas médias e desvios-padrão de 84 éguas da raça Crioula, em diferentes idades (G1, G2 e G3)

\begin{tabular}{lcccc}
\hline \multicolumn{1}{c}{ Variáveis } & G1 $(\mathrm{n}=40)$ & $\mathrm{G} 2(\mathrm{n}=11)$ & $\mathrm{G} 3(\mathrm{n}=33)$ & $\mathrm{p}$ \\
\hline Frequência Cardíaca (bpm) & $52,86 \pm 9,07$ & $51,14 \pm 10,86$ & $54,35 \pm 7,33$ & 0,5367 \\
Duração onda P (ms) & $111,25 \pm 17,955$ & $113,97 \pm 27,04$ & $107,38 \pm 23,53$ & 0,6105 \\
Amplitude onda P (mV) & $0,16 \pm 0,04$ & $0,16 \pm 0,05$ & $0,18 \pm 0,05$ & 0,1454 \\
Intervalo PR (ms) & $237,18 \pm 36,66$ & $264,36 \pm 54,41$ & $235,53 \pm 37,54$ & 0,0980 \\
Complexo QRS (ms) & $110,65 \pm 8,49$ & $101,98 \pm 10,02$ & $100,92 \pm 10,72$ & 0,0002 \\
Amplitude R (mV) & $0,15 \pm 0,10$ & $0,16 \pm 0,13$ & $0,16 \pm 0,13$ & 0,9337 \\
Amplitude S (mV) & $0,12 \pm 0,03$ & $0,13 \pm 0,04$ & $0,11 \pm 0,03$ & 0,5001 \\
Intervalo QT (ms) & $193,11 \pm 30,54$ & $213,21 \pm 34,56$ & $203,21 \pm 25,30$ & 0,0933 \\
ITV & $3,51 \pm 0,61$ & $3,26 \pm 0,59$ & $3,24 \pm 0,52$ & 0,1080 \\
NN médio (ms) & $1156,40 \pm 173,11$ & $1162,60 \pm 244,37$ & $1094,50 \pm 159,32$ & 0,1997 \\
SDNN (ms) & $72,64 \pm 53,11$ & $53,08 \pm 35,47$ & $48,94 \pm 27,78$ & 0,0555 \\
Peso (kg) & $442,70 \pm 53,23$ & $506,40 \pm 60,73$ & $563,80 \pm 47,28$ & $<0,001$
\end{tabular}

$\mathrm{Bpm}=$ batimentos por minuto, $\mathrm{ms}=$ milissegundo, $\mathrm{mV}=$ milivolt, $\mathrm{ITV}=$ índice de tônus vagal, NN médio = média de 15 intervalos R-R normais consecutivos, SDNN = desvio padrão de 15 intervalos R-R normais consecutivos. $\mathrm{p}=$ valores de $\mathrm{p}$ obtidos pelo teste de Tukey. 
não foram detectadas arritmias ou distúrbios de condução nas éguas crioulas aqui estudadas, a despeito da baixa incidência (16\%) observada por Schade et al. (2014) em cavalos crioulos, atribuída à característica da raça. Aliado ao fator racial, acreditamos que os mesmos motivos que levaram a maior prevalência de TS possam estar envolvidos na eliminação dos distúrbios de condução e arritmias dependentes do tônus vagal, a exemplo da bradicardia, bloqueios átrioventriculares e sinus arrest (Marr \& Bowen 2010). No entanto, futuros estudos eletrocardiográficos de longa duração (sistema Holter) poderão auxiliar na detecção de possíveis arritmias cardíacas ao longo de 24 horas (Bakos \& Lohne 2009).

$O$ estudo da conformação das ondas evidenciou maior ocorrência de ondas P de morfologia bífida, complexos QRS do tipo rS e ondas T bifásicas (Fig.1), independente da idade (Quadro 4), corroborando os achados de Schade et al. (2014) no estudo da derivação ápice-base em equinos da raça Crioula. Doze éguas do G1 (24\%) apresentaram ondas T negativas e uma égua do G3 demonstrou morfologia positiva da onda T. A morfologia das ondas eletrocardiográficas depende do sentido e quantidade do impulso elétrico gerado pela despolarização e ou repolarização miocárdica, em relação à polarização dos eletrodos superficialmente posicionados (derivação), resultando em ondas positivas, negativas ou bifásicas/isoelétricas (Tilley \& Goodwin 2002). Neste contexto, a espécie equina apresenta diferenças em sua despolarização em relação a outras espécies (Bonagura \& Reef 2000), resultando em diversas morfologias eletrocardiográficas, as quais também podem ser influenciadas pela raça, idade e sexo (Reef 1992). No entanto, o mesmo comportamento morfológico foi detectados nas diferentes faixas etárias avaliadas, assemelhando-se ao padrão geral da espécie equina para a derivação ápice-base (Patteson 1996), sugerindo a não influência da idade ou da raça Crioula sobre este aspecto eletrocardiográfico.

Com relação a duração e amplitude das ondas e intervalos eletrocardiográficos avaliados, apenas a duração média do complexo QRS foi influenciada pela idade (Quadro 3), com superioridade do grupo G1 em relação ao G2 e G3. Embora haja divergência quanto as faixas etárias estudadas por Schade et al. (2014) em cavalos crioulos, os autores observada influência da idade nas variáveis amplitude da onda P2, duração dos intervalos PR e QT, onda T e complexo QRS, porém, ao contrário do disposto nas éguas do presente estudo, com menor duração do complexo QRS entre1 e 2 anos, quando comparados as demais idades estudadas (3-5, 6-10 e >10 anos). A duração do complexo QRS representa o tempo necessário para a despolarização de toda massa ventricular, inversamente relacionada à frequência cardíaca (Patteson 1996) e diretamente relacionada à massa ventricular (Lightowler et al. 2004). No entanto, não houve diferença entre as frequências cardíacas dos grupos G1, G2 e G3 $(p>0,05)$ que justifique a diferença observada. Na opinião dos autores, a duração do complexo QRS pode ter sido influenciada pelas adaptações autonômicas da prenhes, visto que $100 \%$ das éguas prenhes pertenciam aos grupos G2 e G3, resultando em diminuição deste intervalo quando comparados as éguas não prenhes $(\mathrm{P}<0,05)(\mathrm{Qua}-$ dro 5).

Segundo Tilley \& Goodwin (2002), a inervação autonômica simpática e parassimpática exerce influência direta sobre o automatismo cardíaco e velocidade de condução elétrica, de forma antagônica, na qual o simpático acelera e

Quadro 4. Frequência (\%) de ocorrência das morfologias das ondas $\mathrm{P}$, complexos QRS e ondas $\mathrm{T}$, avaliados nos eletrocardiogramas das 84 éguas da raça Crioula, em diferentes idades (G1, G2 e G3)

\begin{tabular}{|c|c|c|c|c|c|c|}
\hline \multirow[b]{2}{*}{ Grupos } & \multicolumn{3}{|c|}{ Onda T } & \multicolumn{3}{|c|}{ Complexo QRS } \\
\hline & Bifásica & Positiva & Negativa & $\mathrm{rS}$ & Bífida & Única \\
\hline G1( & 70,0 & - & 30,0 & 100 & 82,5 & 17,5 \\
\hline G2 $(1$ & 100 & - & - & 100 & 81,8 & 18,2 \\
\hline G3 (n=33) & 97,0 & 3,0 & - & 100 & 69,7 & 30,3 \\
\hline
\end{tabular}

Quadro 5. Variáveis eletrocardiográficas médias e desvios-padrão de 84 éguas da raça Crioula, segundo a o estado reprodutivo

\begin{tabular}{lccc}
\hline \multicolumn{1}{c}{ Variáveis } & Prenhe & Não Prenhe & $\mathrm{p}$ \\
\hline Frequência Cardíaca (bpm) & $54,84 \pm 8,39$ & $52,11 \pm 8,73$ & 0,1571 \\
Duração onda P (ms) & $110,06 \pm 25,88$ & $110,11 \pm 18,05$ & 0,9927 \\
Amplitude onda P (mV) & $0,17 \pm 0,05$ & $0,17 \pm 0,04$ & 0,5514 \\
Intervalo PR (ms) & $240,94 \pm 43,46$ & $239,52 \pm 38,40$ & 0,8747 \\
Complexo QRS (ms) & $101,96 \pm 9,75$ & $108,36 \pm 10,49$ & 0,0069 \\
Amplitude R (mV) & $0,15 \pm 0,10$ & $0,16 \pm 0,12$ & 0,4755 \\
Amplitude S (mV) & $0,11 \pm 0,03$ & $0,12 \pm 0,03$ & 0,2508 \\
Intervalo QT (ms) & $201,41 \pm 24,54$ & $198,55 \pm 32,86$ & 0,6668 \\
ITV & $3,19 \pm 0,55$ & $3,49 \pm 0,57$ & 0,0191 \\
NN médio (ms) & $1079,40 \pm 157,42$ & $1167,60 \pm 184,91$ & 0,0264 \\
SDNN (ms) & $47,49 \pm 30,16$ & $69,36 \pm 48,89$ & 0,0241 \\
Peso (kg) & $557,40 \pm 56,05$ & $458,60 \pm 61,18$ & $<0,0001$
\end{tabular}

$\overline{\mathrm{Bpm}}=$ batimentos por minuto, $\mathrm{ms}=$ milissegundo, $\mathrm{mV}=$ milivolt, $\mathrm{ITV}=$ índice de tônus vagal, $\mathrm{NN}$ médio = média de 15 intervalos R-R normais consecutivos, SDNN = desvio padrão de 15 intervalos R-R normais consecutivos; $\mathrm{p}=$ valores de $\mathrm{p}$ obtidos pelo teste de $t$.

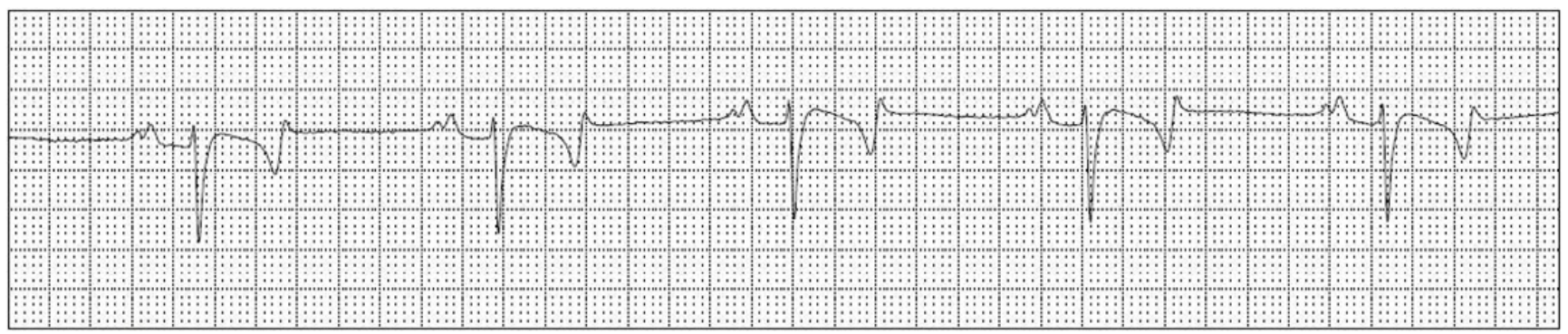

Fig.1. Eletrocardiograma, na derivação ápice-base, sensibilidade $\mathrm{N}$ e velocidade de $25 \mathrm{~mm} / \mathrm{s}$, de uma égua da raça Crioula com 23 anos de idade (G3), evidenciando os aspectos morfológicos de onda P bífida, complexo QRS tipo rS e onda T bifásica, prevalente em todas as idades avaliadas (G1, G2 e G3) 
o parassimpático retarda estas propriedades, podendo alterar a duração das ondas e intervalos eletrocardiográficos. De forma indireta e não invasiva, podemos estimar a participação autonômica simpática e parassimpática, por meio da análise matemática das variações entre as ondas R normais (variabilidade da frequência cardíaca), no domínio do tempo ou da frequência (Acharya et al. 2006). No presente estudo foram utilizados três indicadores de variabilidade da frequência cardíaca no domínio do tempo (ITV, NN médio e SDNN), os quais não diferiram entre as idades estudadas $(p>0,05)$. No entanto, os três indicadores foram superiores nas éguas prenhes em comparação as não prenhes (Quadro 5), sugerindo maior participação simpática e ou menor parassimpática nas éguas prenhes, corroborando a hipótese propostas pelos autores para a diferença observada na duração do complexo QRS. Porém, novos estudos deverão ser realizados para confirmar esta hipótese.

\section{CONCLUSÕES}

Nas condições em que o presente estudo foi realizado, podemos concluir que a idade influenciou apenas na duração do complexo QRS e a prenhes foi capaz de diminuir as variáveis eletrocardiográficas de variabilidade da frequência cardíaca, no domínio do tempo, possivelmente interferindo na avaliação eletrocardiográfica das éguas crioulas avaliadas.

Não foram observadas arritmias ou distúrbios de condução elétrica, com predomínio do ritmo de taquicardia sinusal, compatíveis com os padrões de normalidade para espécie equina, na derivação ápice-base.

\section{REFERÊNCIAS}

ABCCC 2014. História do Cavalo Crioulo, <http://www.racacrioula.com. br/site/content/entidade/cavalo_crioulo.php> Acesso em 4 set. 2014.

Acharya U.R., Joseph K.P., Kannathal N., Lim C.M. \& Suri J.S. 2006. Heart rate variability: a review. Medical and Biological Engineering and Computing 44:1031-1051.

Babusci M. \& López E.F. 2006. Sistema cardiovascular, p.61-85. In: Boffi F.M. (Ed.), Fisiologia del Ejercicio Equino. Inter-Médica, Buenos Aires.

Bakos Z. \& Lohne L. 2009. Reference values of holter electrocardiography in normal horses. West Ind. Vet. J. 9:3-7.

Bello C.A.O., Dumont C.B.S., Souza T.S., Palma J.M., Lima E.M.M., Godoy R.F., Neto G.B.P. \& Meryonne M. 2012. Avaliação eletrocardiográfica de equinos após exercício de polo (baixo handicap). Pesq. Vet. Bras. 32:47-52.

Bonagura J.D. \& Reef V.B. 2000. Doenças Cardiovasculares, p.250-319. In:
Reed \& Bayly W.M. (Eds), Medicina Interna Equina. Guanabara Koogan, Rio de Janeiro.

Corredor M.J.R., Beltran D.D.A. \& Baez C.J.F. 2005. Parámetros electrocardiográficos del equino criollo de la región del piedemonte llanero. Orinoquia. 9:46-55.

Craig N. \& Nunan M. 1998. Entrenamiento del ritmo cardiaco para caballos: perfomance matters. Pty Ltd, Adelaida.

Diniz M.P., Muzzi R.A.L., Muzzi L.A.L. \& Alves G.E.S. 2008. Estudo eletrocardiográfico de eqüinos da raça Mangalarga Marchador. Arq. Bras. Med. Vet. Zootec. 60:536-542.

Diniz M.P., Michima L.E.S. \& Fernandes W.R. 2011. Estudo eletrocardiográfico de equinos de salto sadios. Pesq. Vet. Bras. 31:355-361.

Dumont C.B.S., Leite C.R., Moraes J.M., Alves R.O., Godoy R.F. \& Lima E.M.M. 2010. Parâmetros eletrocardiográficos de equinos Puro Sangue Árabe submetidos a exercício prolongado de enduro. Ciência Rural 40:19661973.

Feitosa F.L. 2004. Semiologia Veterinária: a arte do exame clínico. Roca, São Paulo. 807p.

Fernandes W.R., Larsson M.H.M.A., Alves A.L.G., Fantoni D.T. \& Belli C.B. 2004. Características eletrocardiográficas em equinos clinicamente normais da raça Puro sangue Inglês. Arq. Bras. Med. Vet. Zootec. 56:143149.

Lightowler C., Piccione G., Giudice E., Olmo G.R. \& Cattáneo M.L. 2004. Echocardiography and electrocardiography as means to evaluate potential performance in horses. J. Vet. Sci. 5:259-262.

Loon G.V. \& Patteson M. 2010. Electrophysiology and arrhythmogenesis, p.59-73. In: Marr C.M. \& Bowen M. (Eds), Cardiology of the Horse. 2nd ed. W.B. Saunders, London.

Marr C.M. \& Bowen I.M. 2010. Cardiology of the horse. 2nd ed. W.B. Saunders, London. 294p.

Patteson M.W. 1996. Equine Cardiology. Blackwell Science, Oxford. 254p.

Reef V.B. 1992. Cardiovascular problems associated with poor performance, p.381-410. In: Robinson N.E. (Ed.), Current Therapy in Equine Medicine. 3rd ed. W.B. Saunders, London.

Robertson S.A. 1990. Practical use of ECG in the horse. In Practice. 12:5967.

Schade J., Schade M.F.S. \& Fonteque J.H. 2014. Auscultatory and electrocardiographic characteristic of Crioulo horses. Pesq. Vet. Bras. 34:281-289.

Teixeira P.P., Meirinhos M.L.G., Pádua J.T. \& Vieira D. 2008. Variações cíclicas do cortisol, triidotironina (T3) e tiroxina (T4) no periparto de éguas da raça quarto de milha. Ciênc. Anim. Bras. 9:263-271.

Tárraga K.M. 2002. Avaliação clínica da utilização de bloqueadores farmacológicos no estudo dos parâmetros cardiorrespiratórios e da variabilidade da freqüência cardíaca em modelos experimentais de miocardiopatias em cães. Tese de Doutorado em Clínica Cirúrgica, Faculdade de Medicina Veterinária e Zootecnia, Universidade de São Paulo, São Paulo, SP. 87p.

Tilley L.P. \& Goodwin J.K. 2002. Manual de Cardiologia para Cães e Gatos. 3aㅡ ed. Roca, São Paulo. 489p. 